LBL- -31848

DE93 000565

\title{
Impedance-Based Analysis \\ and Study of Phase Sensitivity \\ In Slow-Wave Two-Beam Accelerators
}

\author{
Jonathan S. Wurtele" \\ Massachusetts Institute of Technology, Cambridge, MA 02139 \\ David H. Whittum** \\ National Laboratory for High Energy Physics (KEK) \\ Tsukuba, 1-1 Oho, Ibaraki, 305 Japan
}

Andrew M. Sessler***

Lawrence Berkeley Laboratory, University of California, Berkeley, California 94720

June 1992

\footnotetext{
- Work supported by the Office of High Energy and Nuclear Physics of the U.S. Department of Energy under Contract No. DE-FG02-91ER40648.

*" Work supported by the Japan Society for the Promotion of Science, the U.S. National Science Foundation and the National Laboratory for High Energy Physics (KEK).

** "Work supported by the Director, Office of Energy Research, Office of High Energy and Nuclear Physics, Division of High Energy Physics, of the U.S. Department of Energy under Contract No. DE-AC0376SF00098
} 


\title{
IMPEDANCE-BASED ANALYSIS \\ AND STUDY OF PHASE SENSITIVITY \\ IN SLOW-WAVE TWO-BEAM ACCELERATORS
}

\author{
Jonathan S. Wurtele* \\ Massachusetts Institute of Technology \\ Cambridge, MA 02139 \\ David H. Whittum"* \\ National Laboratory for High Energy Physics (KEK) \\ Tsukuba, Oho, Ibaraki, 305 Japan \\ Andrew M. Sessler"** \\ Lawrence Berkeley Laboratory \\ University of California \\ Berkeley, CA 94720
}

\begin{abstract}
This paper presents a new formalism which makes the analysis and understanding of both the relativistic klystron (RK) and the standing-wave free-electron laser (SWFEL) two-beam accelerator (TBA) available to a wide audience of accelerator physicists. A "coupling impedance" for both the RK and SWFEWL is introduced, which can include realistic cavity features, such as beam and vacuum ports, in a simple manner. The RK and SWFEL macroparticle equations, which govern the energy and phase evolution of successive bunches in the beam, are of identical form, differing only by multiplicative factors. Expressions are derived for the phase and amplitude sensitivities of the TBA schemes to errors (shot-to-shot jitter) in current and energy. The analysis allows, for the first time, relative comparisons of the RK and the SWFEL TBAs.
\end{abstract}

\footnotetext{
"Supported by the U.S. Department of Energy, Division of Nuclear and High Energy Physics, under contract No. DE-FG02-91ER40648.

* Supported by the Japan Society for the Promotion of Science, the U.S. National Science Foundation and the National Laboratory for High Energy Physics (KEK). *** Supported by the U.S. Department of Energy, Division of Nuclear and High Energy Physics, under contract No. DE-AC-03-SF-00098.
} 


\section{INTRODUCTION}

The context and motivation for this work is the Two-Beam Accelerator (TBA) concept $1,2,3$, which is, in essence, a high efficiency power converter, extracting energy from a low energy high-current electron "drive" beam and depositing it in a high energy electron or positron beam. In a TBA a drive beam of kiloampere current, bunched at centimeter wavelengths, passes through a periodic array of wiggler magnets, which extract the beam energy through a Relativistic Klystron (RK) or a Free-Electron Laser (FEL); at the same time, the beam passes through induction cells which replenish the beam energy, as seen in Figs. 1 and 2. The high power microwaves produced in the interaction region are periodically extracted and fed into a high gradient structure, where they accelerate the second beam.

The TBA configuration of present interest, the Standing-Wave Free-Electron Laser TBA (SWFEL/TBA), has grown out of a number of theoretical and conceptual refinements, including considerations of microwave extraction and phase and amplitude control.4.5 In the original configuration, a large amplitude microwave signal propagates with the drive beam over the entire length of the accelerator. In each FEL section the microwave power was produced and extracted, by septa, in such a way that the total power remains roughly constant. This design allowed for the continuous longitudinal bunching of the electron beam through each FEL section. There were drawbacks to the scheme, however. The microwaves had to be transported across the induction unit gaps. Theoretical investigations discovered that while the longitudinal beam motion was stable, the rf phase shift produced by the FEL interaction had an undesirable sensitivity to shot-to-shot jitter in the induction units and in the beam current. It was proposed that the $\mathrm{rf}$ power be fully extracted at the end of each wiggler section, before reacceleration, thereby limiting the total accumulated phase shift in the wave, and reducing the sensitivity to jitter in the system parameters.

Further considerations of the rf extraction mechanism led to the development of the SWFEL 6 in which the power is produced in a series of uncoupled cavities (the $\mathrm{rf}$ is cut off between the cavities), each of which is of order one wiggler oscillation in length. The FEL thus operates as a standing-wave device. The propagating beam provides the only coupling between the cavities. Numerical studies 7,8 have examined the phase sensitivity and longitudinal particle stability in the standing wave FEL in some detail. 


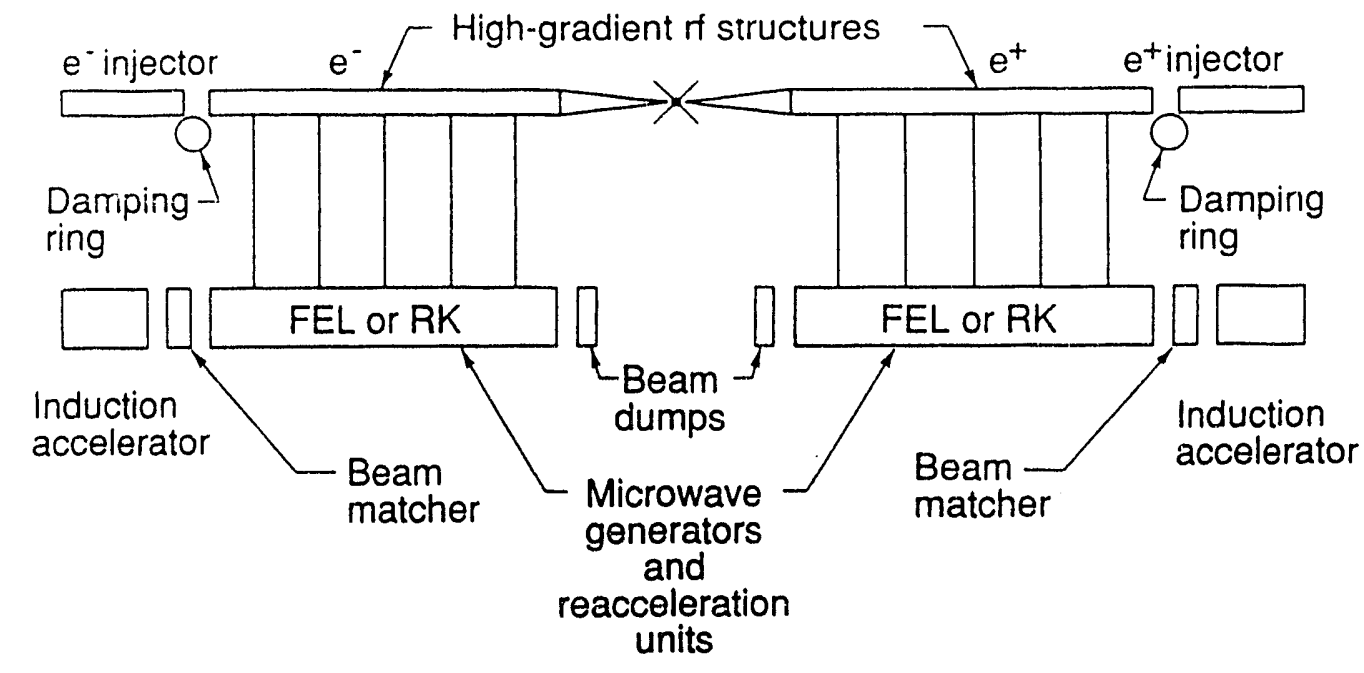

Fig. 1. A schematic of the structure of a TBA. The cavities can either be those of a relativistic klystron (RK), or those of a Standing-Wave FEL (SWFEL) in which case there is a wiggler magnetic field passing through the cavities.

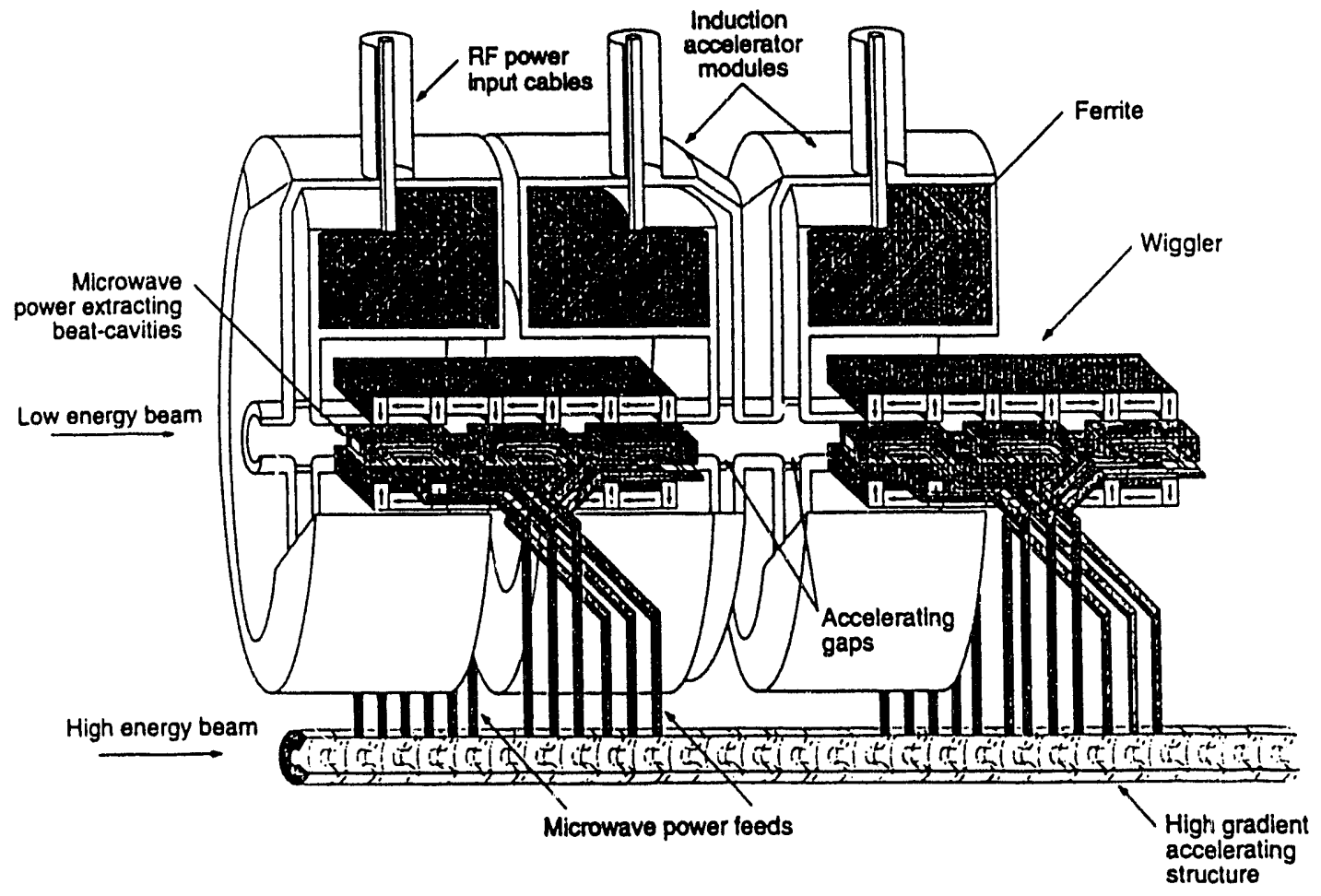

Fig. 2. A schematic of one superperiod of the Standing-Wave FEL TBA. Microwaves are produced by a low energy drive beam and fed into a high gradient structure to accelerate a high energy. 
In all of the above work, the extraction units were taken as FELs. Alternatively, of course, it is possible to consider a Relativistic Klystron TBA (RK/TBA). This approach has been developed by the CERN Group. ${ }^{9}$ Because it has been demonstrated experimentally that high power can be extracted from an $\mathrm{RK}^{10}$, as well as from an FEL 11 , both of these approaches are attractive. In fact, the standing wave FEL has many similarities to the relativistic klystron, the main difference between them being that the FEL produces power through the coupling of the transverse wiggle oscillation with the transverse electric field, while the klystron couples the longitudinal component of the electric field. Until now, no serious comparisons of these two approaches has been made. In fact, not even the formal framework in which such comparisons can be made has been developed. It is the purpose of this paper to set down such a framework.

In Section 2, we develop a formalism that allows comparisons of the RK and SWFEL TBAs. In Section 3 we evaluate phase sensitivities in the two approaches to a TBA. Section 4 contains discussion and conclusions.

\section{FORMALISM}

In this section we derive equations describing the coupling of beam electrons to cavity modes. We first decompose the vector potential in the Lorentz gauge,

$$
\vec{A}=\frac{m c^{2}}{e} \sum_{\alpha} q_{\alpha}(t) \vec{a}_{\alpha}(\vec{r})
$$

where $\alpha$ is the mode index, $q_{\alpha}$ is the dimensionless mode amplitude and $\vec{a}_{\alpha}$ gives the spatial dependence of the mode. The electron mass is $m$, the speed of light is $c$ and the electron charge is $-e$. The mode normalization is

$$
\int_{V} d^{3} r^{\prime} \vec{a}_{\alpha}(\vec{r}) \cdot \vec{a}_{\alpha}^{*}(\vec{r})=V
$$

with $V$ the cavity volume.

Maxwell's equations reduce to the well-known form

$$
\left(\frac{\partial^{2}}{\partial t^{2}}+\frac{\omega_{\alpha}}{Q_{\alpha}} \frac{\partial}{\partial t}+\omega_{\alpha}^{2}\right) q_{\alpha}(t)=\frac{4 \pi x}{m c} \frac{1}{V} \int d^{3} r^{\prime} \vec{J}\left(\vec{r}^{\prime}, t\right) \cdot \vec{a}_{\alpha}\left(\vec{r}^{\prime}\right)
$$


where the integral is over the cavity volume. We consider the interaction of the beam with a single cavity mode with a very high $Q$, and make an eikonal approximation, $q(t)=\mathfrak{R}\left\{b e^{i \varphi} e^{-i \omega t}\right\}$, where the phase $\varphi$ and the amplitude $b$ vary slowly on the time scale of the mode period. In terms of $b$, the energy stored per unit length is

$$
U=\frac{1}{8 \pi}\left[\frac{\omega^{2} h w}{c^{2}}\right] \frac{m^{2} c^{4} b^{2}}{e^{2}},
$$

where $h$ and $w$ are the height and width of the cavity.

We will consider two cases: (1) coupling to a TE mode through the transverse current induced by a magnetic wiggler (FEL) and (2) coupling to a TM mode through the axial current (RK). In each case the coupling depends on the phase $\psi=\varphi+\theta$ of an electron's motion relative to the phase of the cavity fields. Here the phase $\theta$ is a particle variable. For an FEL this phase is given by

$$
\theta=\left(k_{\omega}+k_{z}\right) z-\omega t
$$

where $k_{w}$ is the wiggler wavenumber and $k_{z}$ is the axial wavenumber for the forward-going component of the cavity mode. For a steadystate klystron this phase is

$$
\theta=k_{z} z-\omega t-\theta_{r}
$$

where we have introduced the phase $\theta_{r}$, that of a reference particle. Typically klystrons operate with $k_{z}=0$, in a nearly single mode cavity. The SWFEL, on the other hand, operates in a highly overmoded cavity.

An important distinction between the SWFEL and the RK is that Eq. (5) defines a synchronous energy in terms of the system parameters, while Eq. (6), for the RK, only relates the phase of a particle to a reference phase and does not define a synchronous energy. The RK, therefore, can be operated at any energy (even $\mathrm{GeV}$ energies are possible), whereas the SWFEL requires a low energy (of order ten $\mathrm{MeV}$ ) for resonance at microwave frequencies with reasonable wiggler parameters.

In terms of these variables the field equations in a given cavity may be written as 


$$
\frac{\partial}{\partial s} b e^{i \varphi}=i c \frac{1}{\eta} \frac{r}{Q} \frac{I}{I_{A}}\left\langle e^{-i \theta}\right\rangle
$$

where $s=v_{z} t-z$, with $v_{z}$ the beam velocity. $I$ is the average beam current, $I_{A}=m c^{3} / e \sim 17 \mathrm{kA}$, and the brackets indicate an average over a beam slice. The factor $\eta$ depends on the kind of coupling. For an RK $\eta=2$, while for an FEL, $\eta=a_{w} / 2 \gamma$, with $\gamma$ the Lorentz factor, and $a_{w}$ the wiggler parameter.

The shunt impedance per unit length $r$ is given by 6

$$
\frac{r}{Q}=\left.\left.\frac{4 \pi}{V L \omega}\right|_{-L / 2} ^{+L / 2} d z \frac{\vec{v}(z)}{v_{z}} \bullet \vec{a}(z) \exp \left(-\frac{i \omega z}{v_{z}}\right)\right|^{2},
$$

where $L$ is the cavity length. The SWFEL typically operates in the $\mathrm{TE}_{01 \mathrm{p}}$ mode of a rectangular cavity of width $w$ and height $h$, so that

$$
\frac{r}{Q}=\frac{Z_{0}}{8 \pi} \frac{\lambda}{h w}\left(\frac{a_{w}}{\gamma}\right)^{2}\left(\frac{\sin \chi}{\chi}\right)^{2},
$$

where $Z_{0}=4 \pi / c$ (377 $\Omega$ in MKS), $\lambda$ is the free-space wavelength and $\chi=$ $\left(\omega L / v_{z}-p \pi-k_{w} L\right) / 2$ is the effective transit angle. For an RK operating in the $\mathrm{TM}_{\mathrm{m} 1 \mathrm{p}}$ mode,

$$
\frac{r}{Q}=\frac{Z_{0}}{4 \pi} \frac{\lambda}{h w}\left\{\frac{k_{x}^{2}+k_{y}^{2}}{k_{x}^{2}+k_{y}^{2}+k_{z}^{2}}\right\}\left(\frac{\sin \chi}{\chi}\right)^{2},
$$

where the transit angle is $\chi=\left(p \pi+\omega L / v_{z}\right) / 2$. The coupling in the SWFEL is from the interaction of the wiggling velocity imparted to the beam by the wiggler and the transverse field of a TE mode, while the RK generates a shunt impedance from the axial coupling of the beam to the z-component of the electric field of a TM mode.

To complete the formulation, equations are required for the particle motion. It is convenient to linearize about the reference energy, so that the dynamical variables are $\theta$ and $\delta \gamma=\gamma-\gamma_{r^{\prime}}$ where $\gamma_{r}$ is the resonant $\gamma$ in the case of the FEL, or in the case of an RK, a reference $\gamma$. The phase evolution is found from Eqs. (5) and (6), so that ${ }^{6}$ 


$$
\begin{gathered}
\frac{d \theta}{d z}=2 \kappa \frac{\delta \gamma}{\gamma_{r}} \\
\frac{d \delta \gamma}{d z}=-\eta \frac{\omega}{c} b \sin (\theta+\varphi)-\frac{e E_{z}}{m c^{2}}
\end{gathered}
$$

The constant $\kappa=\omega\left(1+a_{w}^{2} / 2\right) / 2 c \gamma^{2}$ for an FEL, while $\kappa=\omega / 2 c \gamma^{2}$ for the RK. Equations (7), (11), and (12) describe the self-consis ient evolution of the beam and the cavity fields. The SWFEL and $R K$ are distinguished only through the values of $\eta, \kappa$ and $r / Q$. These equations can be obtained from a continuum limit of a discrete cavity analysis by assuming that the energy and phase change in a given cavity are small. Using Eq. (4) it is straightforward to check that these equations conserve energy.

We will consider an equilibrium (no $z$-dependence of the dependent variables) of a well-bunched constant-current beam, described by (fixed) parameters $\alpha, \beta$ (where the amount of detuning is characterized by $\beta$ )

$$
\theta_{0}(s)=\alpha+\beta s
$$

Substituting Eq. (13) in Eq. (7) one obtains the equilibrium field,

$$
\begin{aligned}
b_{0}(s)^{2}-b_{0}(0)^{2} & = \\
2 & \frac{\varepsilon b_{0}(0)}{\beta}\left[\cos \left(\alpha+\varphi_{0}\right)-\cos \left(\alpha+\varphi_{0}+\beta s\right)\right]+\left(\frac{2 \varepsilon}{\beta}\right)^{2} \sin ^{2}\left(\frac{\beta s}{2}\right) .
\end{aligned}
$$

Equation (12) and the equilibrium condition require a reaccelerating field given by

$$
\frac{e E_{z}}{m c^{2}}=-\eta_{i} \frac{\omega}{c}\left[\tilde{b}_{0 r}(0) \sin (\alpha+\beta s)+\tilde{b}_{0 i}(0) \cos (\alpha+\beta s)+\frac{\varepsilon}{\beta} \sin (\beta s)\right] \text {. }
$$

Here we abbreviate $\tilde{b}=b_{0} e^{i \varphi_{0}}$ and

$$
\varepsilon=c \frac{1}{\eta} \frac{r}{Q} \frac{I}{I_{0}}
$$

The important results of this section are the equations describing an equilibrium (Eqs. 13 and 14), which allow us to study, as 
we shall in the next section, sensitivities to errors in beam current or energy, and the expression for the field (Eq. 7) in terms of a generalized coupling impedance defined in Eqs. (9) and (10).

\section{RF AMPLITUDE AND PHASE SENSITIVITY TO JITTER}

Next we consider the perturbation to the equilibrium resulting from an error in the initial beam energy $m c^{2} \Delta \gamma$ and an error $\Delta I$ in the beam current. The errors in amplitude and phase will be found by evaluating the perturbed field, $b_{1}(s, z)=b-b_{0}$.

The evolution of the perturbed phase $\theta(s, z)=\theta_{0}(s)+\theta_{1}(s, z)$ is governed by Eqs. (11) and (12), which combine to give

$$
\frac{d^{2} \theta_{1}}{d z^{2}}+\Omega^{2}(s) \theta_{1}=-\frac{2 \eta \kappa \omega}{c \gamma}\left\{b_{i 1}^{-} \cos \theta_{0}+b_{r 1}^{-} \sin \theta_{0}\right\}
$$

where the synchrotron period is

$$
\Omega^{2}(s)=\frac{2 \eta \kappa \omega}{\gamma} b_{0} \cos \psi_{0} \approx \Omega^{2}(0)-\frac{4 \varepsilon \kappa \eta \omega}{\gamma \beta c} \sin ^{2} \frac{\beta s}{2} .
$$

The perturbed eikonal, $\tilde{b_{1}}$, is determined from Eq. (6), to be:

$$
\frac{\partial}{\partial s} \tilde{b_{1}}=i \varepsilon_{1} e^{-i \theta_{0}}+\varepsilon_{0} e^{-i \theta_{0}} \theta_{1}
$$

Here $\varepsilon_{1}=(\operatorname{cr} \Delta I) /\left(\eta Q I_{A}\right)$, and $\varepsilon_{0}=\left(\operatorname{cr} I_{o}\right) /\left(\eta Q I_{A}\right)$. Energy errors are included in the choice of initial condition for $\dot{d} \theta_{1} / d z(z=0)$. Errors in energy due to jitter in $\varepsilon$ (due to loading of the induction cell circuit) are ignored.

Equation (19) may be solved up to quadrature and substituted in Eq. (17) to obtain

$$
\begin{aligned}
& \frac{d^{2} \theta_{1}}{d z^{2}}+\Omega^{2}(s) \theta_{1}= \\
& -\int_{0}^{s} \mu_{1} \cos \left\{\beta\left(s-s^{\prime}\right)\right\} d s^{\prime}-\int_{0}^{s} \mu_{0} \sin \left\{\beta\left(s-s^{\prime}\right)\right\} \theta_{1}\left(s^{\prime}, z\right) d s^{\prime},
\end{aligned}
$$


where we abbreviate $\mu_{\left(\begin{array}{l}1 \\ 0\end{array}\right)}=\frac{2 \eta \kappa}{\gamma} \frac{\omega}{c} \varepsilon_{\left(\begin{array}{l}1 \\ 0\end{array}\right)}$. Note that the driving term due to the current error ( $\mu_{1}$ term) is independent of $z$.

In order to solve Eq. (20), we set $\theta_{1}=\delta+\tilde{\theta}_{1}$, where $\delta$, the phase error due to current jitter, is independent of $z$, and evolves according to:

$$
\begin{aligned}
& \Omega^{2}(s) \delta(s)= \\
& -\int_{0}^{s} \mu_{1} \cos \left\{\beta\left(s-s^{\prime}\right)\right\} d s^{\prime}-\int_{0}^{s} \mu_{0} \sin \left\{\beta\left(s-s^{\prime}\right)\right\} \delta\left(s^{\prime}\right) d s^{\prime} .
\end{aligned}
$$

As expected, $\delta(0)=0$ and, when $\mu_{1}=0, \delta(s)=0$ for all $s$. Equation (20) then reduces to

$$
\frac{d^{2} \tilde{\theta}_{1}}{d z^{2}}+\Omega^{2}(s) \tilde{\theta}_{1}=-\int_{0}^{s} \mu_{0} \sin \left\{\beta\left(s-s^{\prime}\right)\right\} \tilde{\theta}_{1}\left(s^{\prime}, z\right) d s^{\prime}
$$

an equation of the "beam break-up" form. Note that the driving term on the right represents the feedback experienced by the perturbed beam.

To obtain an upper bound on the effect of this driving term, consider the problem with $\Omega(s)=\Omega(0)$. (This will lead to an upper bound, since $\Omega(s)$ increases with $s$, providing ever stronger focusing). The problem is then formally equivalent to the problem of "headtail" beam break-up. ${ }^{12}$ In the limit of large $z$, the asymptotic form is

$$
\tilde{\theta}_{1} \approx \exp \left\{i \Omega(0) z+\frac{3}{2}\left(\frac{\mu_{0} \beta z s^{2}}{\Omega(0)}\right)^{1 / 3} e^{i \pi / 6}\right\} \text {. }
$$

Evidently, feedback is negligible when $\Omega(0) \gg \mu_{0} \beta z s^{2}$. For typical parameters, this driving term is indeed negligible, and the particle n.otion is well-described by a free synchrotron oscillation (i.e., the motion of a "test particle"). In this case, the solution to Eq. (23) is

$$
\tilde{\theta}_{1}=\frac{1}{\Omega(s)}\left(\frac{d \theta}{d z}\right)_{0} \sin \Omega(s) z=2 \kappa \frac{\Delta \gamma}{\gamma_{r} \Omega(s)} \sin \Omega(s) z .
$$

An analogous argument for $\delta(s)$ in Eq. (21) leads to: 


$$
\delta(s)=\frac{\mu_{1} \sin \beta s}{\Omega^{2}(s) \beta},
$$

Employing Eqs (24) and (25), the error in the cavity field is obtained, from Eq. (19), as

$$
\begin{aligned}
& \tilde{b}_{1}(s, z)=\varepsilon_{0} \int_{0}^{s} d s^{\prime} \theta_{1}\left(s^{\prime}, z\right) e^{-i \theta_{0}\left(s^{\prime}\right)}+i \varepsilon_{1} \int_{0}^{s} d s^{\prime} e^{-i \theta_{0}\left(s^{\prime}\right)} \\
& =\varepsilon_{0} \int_{0}^{s} d s^{\prime}\left\{\left[2 \kappa \frac{\Delta \gamma \sin \left(\Omega\left(s^{\prime}\right) z\right)}{\gamma} \Omega\left(s^{\prime}\right)\right]+\delta\left(s^{\prime}\right)\right\} e^{-i \theta_{0}\left(s^{\prime}\right)}+i \varepsilon_{1} \int_{0}^{s} d s^{\prime} e^{-i \theta_{0}\left(s^{\prime}\right)} .
\end{aligned}
$$

Expressions for the amplitude $\left(b_{1}\right)$ and phase $\left(\phi_{1}\right)$ errors caused by the energy error $m c^{2} \Delta \gamma$ and the current error $\Delta I$ may be obtained from Eq. (26). It suffices, in order to get a bound, to replace $\Omega(s)$ with $\Omega(0)$ in the $\kappa$-term. One finds after much algebra (and taking the equilibrium bunch length, $s$, such that $\beta s=-\pi)$ :

$$
\begin{gathered}
\frac{b_{1}}{b_{0}}=-\frac{\mu_{1} g_{r}}{2 \beta \Omega^{2}(0)}+\frac{\varepsilon_{1}}{\varepsilon_{0}} \\
\phi_{1}=-2 \kappa \frac{\Delta \gamma \sin \Omega_{0}(0)}{\gamma}+\frac{\mu_{1} g_{i}}{2 \beta \Omega^{2}(0)} .
\end{gathered}
$$

The factors $g_{r}$ and $g_{i}$ are

$$
\begin{aligned}
& g_{r}=\int_{0}^{\pi} d y \frac{\sin y \cos y}{\left[1+\Delta \sin ^{2} y / 2\right]}, \\
& g_{i}=\int_{0}^{\pi} d y \frac{\sin ^{2} y}{\left[1+\Delta \sin ^{2} y / 2\right]},
\end{aligned}
$$

where

$$
\Delta=\left[\frac{\Omega^{2}\left(s_{F}\right)-\Omega^{2}(0)}{\Omega^{2}(0)}\right] .
$$

The factors $g_{r}$ and $g_{i}$ are of order unity; they may be easily evaluated numerically. 


\section{DISCUSSION AND CONCLUSIONS}

The sensitivities of the RK/TBA and an SWFEL/TBA can now be compared using the results of the last section (Eqs. (27) through (30)). The first thing to observe is that the dependence upon current error, $\Delta I$, is not excessive, nor is it very different for the RK and the SWFEL. This source of sensitivity must, and can, be controlled in either device.

The dependence upon energy errors, $m c^{2} \Delta \gamma$, is much more severe and it is different between the two devices. Note that it only affects $\varphi_{1}$ (and not the amplitude $b_{1}$ ). From Eq. (28) and the expressions for $\kappa$ (just after Eq. (2)) we obtain, for the energy dependence,

$$
\begin{aligned}
& \varphi_{1}=-\left(\frac{\omega}{c \gamma^{2}}\right)\left(\frac{\Delta \gamma}{\gamma}\right) \frac{\sin \Omega(0) z}{\Omega(0)} ; \quad R K, \\
& \varphi_{1}=-\frac{\omega}{c \gamma^{2}}\left(1+a_{w}^{2} / 2\right)\left(\frac{\Delta \gamma}{\gamma}\right) \frac{\sin \Omega(0) z}{\Omega(0)} ; \quad \text { SWFEL. }
\end{aligned}
$$

The initial synchrotron period, given by Eq. (18), can be inserted to obtain the amplitude of phase error:

$$
\text { amplitude of } \varphi_{1}=-\left\{\frac{1}{\left(b_{0}(0) \cos \theta_{0} \varphi_{0}\right)}\right\}^{1 / 2}\left(\frac{\Delta \gamma}{\gamma}\right)\left\{\begin{array}{c}
\left(\frac{1}{2 \gamma}\right)^{1 / 2} ; R K, \\
2^{1 / 2}\left(\frac{1+a_{w}^{2} ; 2}{a_{w}}\right)^{1 / 2} ; \text { SWFEL } .
\end{array}\right.
$$

Taking $b_{0}(0) \cos \left(\theta_{0} \varphi_{0}\right)$ the same for the two devices, we see that the factor $\left(\frac{1}{2 \gamma}\right)^{1 / 2}$, for the RK, is replaced by $(2 \sqrt{2})^{1 / 2}$ for the SWFEL. (We have minimized the coefficient by taking $a_{w}=\sqrt{2}$.)

We see that, as a rule of thumb, the RK is roughly two times less sensitive to energy errors at a rather low energy than is the SWFEL. However, we must remember that the RK will have more severe wake-field effects than the SWFEL since it necessarily consists of smaller structures.

On the other hand, it is possible to operate the RK at a very high energy since no resonance condition must be satisfied (as in the 
SWFEL). At large $\gamma$, the sensitivity to energy errors, $m c^{2} \Delta \gamma$, is very much less in the RK than in the SWFEL. Successful operation of an RK of high energy will, however, depend on acceleration of an intense bunched beam from a low energy, during which process phase errors may yet accumulate. Indeed, accelerating the drive beam of an RK to high energies, while maintaining its phase insensitivity is an important challenge for such a device, and remains to be analyzed.

In summary, we have developed formulas for the sensitivities of the SWFEL/TBA and the RK/TBA. The parameter which characterizes the sensitivity is displayed, and this has allowed us to compare the two schemes. It is shown that there is no vast difference between the two appruaches, although the RK/TBA is less sensitive at high energies. The choice between them will probably be made on the basis of such issues as ease of construction, cost, and beam breakup limits (BBU).

In this last regard, note that the RK/FEL consists of single mode structures (as contrasted with the overmoded SWFEL/TBA), and, hence, will have a lower BBU limit or, equivalently, will be limited to operation at lower frequencies. Thus, high frequency operation (well above $10 \mathrm{GHz}$ ) will require the SWFEL/TBA; if one desires to operate at lower frequencies (well below $10 \mathrm{GHz}$ ) then the $\mathrm{RK} / \mathrm{TBA}$ with its reduced sensitivities is the scheme of choice.

Finally, we note that the formalism which has been developed allows the input of a coupling impedance into the SWFEL and, therefore, the introduction of the features of a realistic cavity. It is interesting, but really obvious, that the properties of the cavity are included in just a single impedance function. We thus have the capability to analyze the performance of a SWFEL employing coupling impedances obtained by various electrodynamic codes such as SUPERFISH or MAFIA. In short, we have put the analysis of the standing-wave free-electron laser on the same footing as tha: of the relativistic klystron.

\section{REFERENCES}

1. A.M. Sessler, in Laser Acceleration of Particles, P.J. Channell ed., AIP Proceedings No. 91, p. 163, New York, (1982).

2. D.B. Hopkins, A. M. Sessler, and J. S. Wurtele, Nucl. Instr. and Meth. in Phys. Res. 228, 15 (1984). 
3. E. Sternbach and A. M. Sessler, Nucl. Instr. and Methods in Phys. Res. A250, 464 (1986).

4. A.M. Sessler, E. Sternbach and J.S. Wurtele, Nucl. Instr. and Methods in Phys. Res. B40/41, 1064 (1989).

5. A.M. Ses'er, D.H. Whittum and J.S. Wurtele, Proc. of the XIV Intern. Conf. on High Energy Accel., Tsukuba, Part. Accel. 31, 69 (1990).

6. A.M. Sessler, et al., Nucl. Instr. and Methods in Phys. Res. A306, 592 (1991).

7. W.M. Sharp, et al., Linear Accelerator Conf, Albuquerque, Los Alamos National Laboratory Report LA-12004-C, 656 (1990).

8. W.M. Sharp, et al., Intense Microwave and Particle Beams II, O/E LASE' 91, Los Angeles, in Proc. Intern. Soc. Opt. Eng. (SPIE) (1991).

9. S. Van Der Meer, Particle Accelerators 30, 127 (1990).

10. M.A. Allen et al., Phys. Rev. Lett. 63, 2472 (1989).

11. T. Orzechowski et al., Phys. Rev. Lett. 57, 2172 (1986).

12. A.W. Chao, B. Richter, and C.Y. Yao, XIth Int. Conf. on High Energy Accelerators, p. 597 (1980); Nucl. Instrum. Methods 178, 1 (1980). 

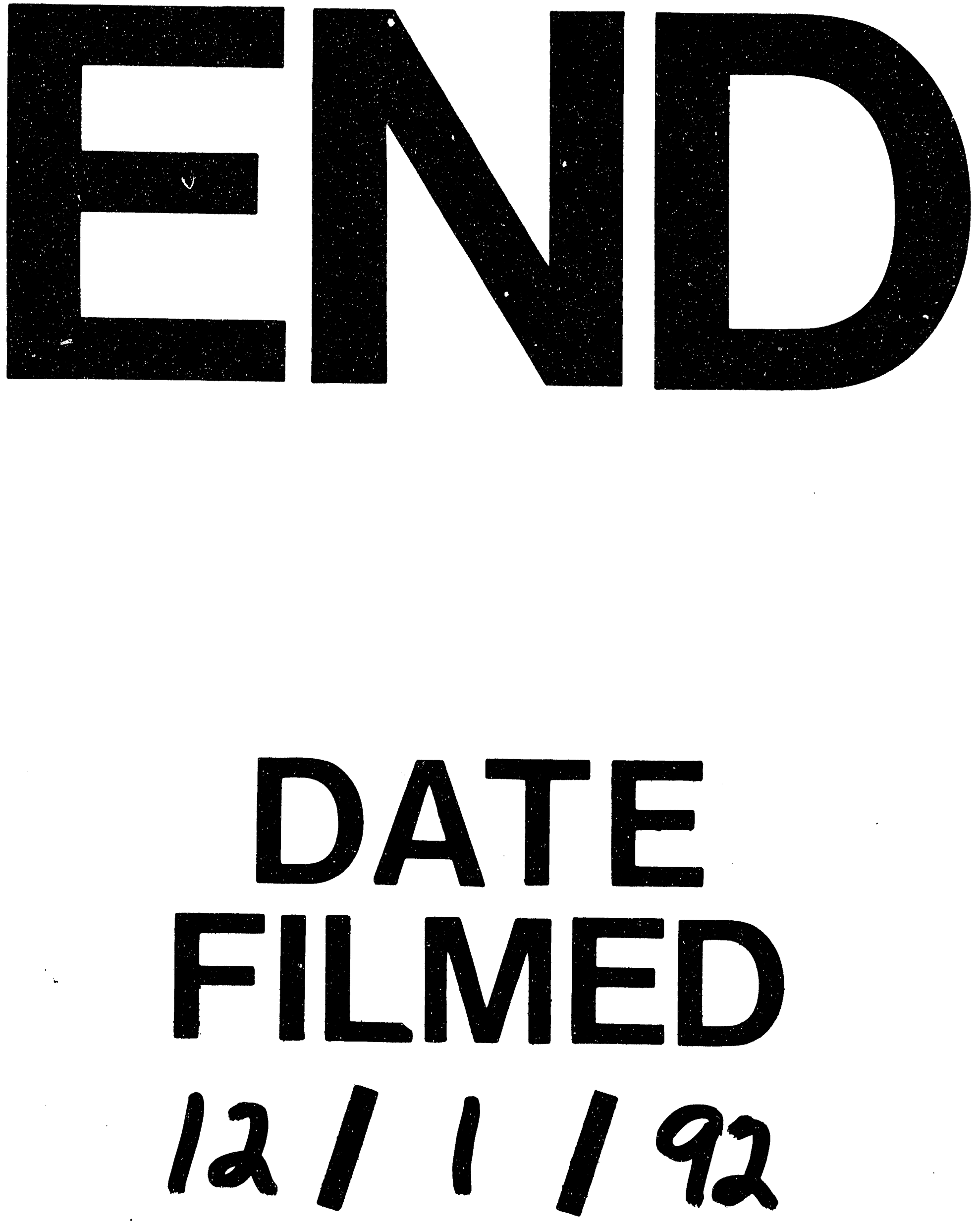
